\title{
Boundary Wess-Zumino-Novikov-Witten Model from the Pairing Hamiltonian
}

\author{
Tigran A. Sedrakyan and Victor Galitski \\ Joint Quantum Institute and Condensed Matter Theory Center, \\ Department of Physics, University of Maryland, College Park, MD 20742, USA
}

(Dated: November 29, 2018)

\begin{abstract}
Correlation functions in the Wess-Zumino-Novikov-Witten (WZNW) theory satisfy a system of Knizhnik-Zamolodchikov (KZ) equations, which involve constants of motion of an exactly solvable model, known as Gaudin magnet. We show that modified KZ equations, where the Gaudin operators are replaced by constants of motion of the closely related pairing Hamiltonian, give rise to a deformed WZNW model that contains terms breaking translational symmetry. This boundary WZNW model is identified and solved. The solution establishes a connection between the WZNW model and the pairing Hamiltonian in the theory of superconductivity. We also argue and demonstrate on an explicit example that our general approach can be used to derive exact solutions to a variety of dynamical systems.
\end{abstract}

PACS numbers: 71.10.Pm, 74.20.Fg, 02.30.Ik

\section{INTRODUCTION}

The Wess-Zumino-Novikov-Witten (WZNW) model plays an important role in physics. Historically, the $S U(2)$ version of the model with topological coupling $k=1$ was used to describe low-energy dynamics of a onedimensional spin- $1 / 2$ Heisenberg antiferromagnet. At higher integer couplings, $k$, it describes quantum critical points in the parameter space of quantum antiferromagnetic spin-S chains, with $S=k / 2 \underline{1}$. Many other applications of the WZNW model have emerged in various contexts lately (see, e.g., Ref. [2]). The Lagrangian formulation of the theory is given by a non-linear sigmamodel defined in the Euclidean space by the action ${ }^{3}$

$$
\begin{aligned}
S_{W Z N W}(g)= & \frac{k}{16 \pi} \int_{S^{2}} d z d \bar{z} \operatorname{tr}\left[\partial_{a} g^{\dagger} \partial^{a} g\right] \\
& -\frac{i k}{24 \pi} \int_{B^{3}} d^{3} x \varepsilon^{\mu \nu \rho} \operatorname{tr}\left[g^{\dagger} \partial_{\mu} g g^{\dagger} \partial_{\nu} g g^{\dagger} \partial_{\rho} g\right]
\end{aligned}
$$

where integration in the second topological Wess-Zumino term is over a three-dimensional ball, $x=(z, \bar{z}, \xi) \in B^{3}$, whose boundary at $\xi=0$ is the two-dimensional sphere, $S^{2}=\partial B^{3}$, which corresponds to a compactified complex plane parametrized by $(z, \bar{z})$ and $g(z, \bar{z}) \in S U(2)$. The integer parameter $k$ in Eq. (11) is the level of the corresponding conformal field theory (CFT). The WZNW action is invariant under conformal and non-Abelian current algebras. The current algebra transformations have a chiral structure, i.e. they act on the group element $g(z, \bar{z})$ as $g^{\prime}(z, \bar{z})=\mathcal{U}(z) g(z, \bar{z}) \overline{\mathcal{U}}(\bar{z})$. Here $\mathcal{U}(z)$ and $\overline{\mathcal{U}}(\bar{z})$ are independent elements of the group $S U(2)$. This property allows to study the holomorphic ( $z$-dependent) and antiholomorphic ( $\bar{z}$-dependent) sectors of the model separately (below, we focus on the holomorphic sector).

The $N$-point correlators of primary fields, $G\left(z_{1} \ldots z_{N}\right)=\left\langle\phi\left(z_{1}, \bar{z}_{1}\right) \ldots \phi\left(z_{N}, \bar{z}_{N}\right)\right\rangle_{S_{\mathrm{WZNW}}}$, sat- isfy the Knizhnik-Zamolodchikov (KZ) equations ${ }^{4}$,

$$
\left[(k+2) \partial_{z_{l}}-\hat{H}_{l}^{G}\right] G\left(\left\{z_{l}\right\}\right)=0,
$$

with

$$
\hat{H}_{l}^{G}=\sum_{l \neq l^{\prime}} w\left(z_{l}, z_{l^{\prime}}\right) \hat{\mathbf{S}}_{l} \cdot \hat{\mathbf{S}}_{l^{\prime}}
$$

$l, l^{\prime}=1,2, \ldots N$, and $w_{l, l^{\prime}}=\left(z_{l}-z_{l^{\prime}}\right)^{-1}$. Here $\hat{\mathbf{S}}_{l}=$ $\left(\hat{S}_{l}^{1}, \hat{S}_{l}^{2}, \hat{S}_{l}^{3}\right)$ and $\hat{S}_{l}^{a}$ are $\mathrm{SU}(2)$ generators. Amazingly, operators $\hat{H}_{l}^{G}$ in Eq. (2) are formally equivalent to the integrals of motion of a seemingly unrelated Gaudin magnet mode $\mathrm{l}^{5,6}$. The Gaudin magnet represents a quantum spin Hamiltonian, with effective long-range interactions between spins, which is exactly solvable "by design." Its Hamiltonian can be represented as a linear combination of the mutually commuting integrals of motion, $\left[\hat{H}_{l}^{G}, \hat{H}_{l^{\prime}}^{G}\right]=0, \forall l, l^{\prime}$ as follows:

$$
\hat{\mathcal{H}}=2 \sum_{l} z_{l} \hat{H}_{l}^{G} .
$$

One general question that we formulate in this paper is whether it is possible to derive deformed WZNW models, whose correlators satisfy modified KZ equations (2) with a different set of operators $\hat{H}_{l}$. Below, we answer this question in the affirmative by providing an example of this "reverse engineering approach" and finding a boundary WZNW model, which corresponds to the operators $\hat{H}_{l}$ representing the integrals of motion of the discrete pairing Hamiltonian (Richardson model) $\underline{\underline{6}} \underline{-13,15} \underline{-18}$ closely related to the Gaudin magnet. It descends from the familiar BCS Hamiltonian

$$
\hat{\mathcal{H}}_{\mathrm{RBCS}}=\sum_{l, s= \pm} z_{l} \hat{c}_{l s}^{\dagger} \hat{c}_{l s}-\lambda \sum_{l, l^{\prime} S} \hat{c}_{l+}^{\dagger} \hat{c}_{l-}^{\dagger} \hat{c}_{l^{\prime}-} \hat{c}_{l^{\prime}+}
$$

where $\hat{c}_{l s}^{\dagger}$ and $\hat{c}_{l s}$ are fermion creation/annihilation operators corresponding to a single-particle state $|l\rangle$ with 
energies $z_{l}$ and spin $s= \pm$. If $\lambda>0$, the ground state is a superconductor with all fermions paired $\frac{19}{19}$. Then, the operators $\hat{c}_{l+}^{\dagger} \hat{c}_{l-}^{\dagger}, \hat{c}_{l^{\prime}-} \hat{c}_{l^{\prime}+}$, and $\sum_{s}\left(\hat{c}_{l s}^{\dagger} \hat{c}_{l s}-1 / 2\right) / 2$ become algebraically equivalent to the Pauli matrices $\hat{\sigma}_{l}^{\dagger}$, $\hat{\sigma}_{l}^{-}$, and $\hat{\sigma}_{l}^{3}$ (Anderson pseudospins). The corresponding spin Hamiltonian is the integrable Richardson model, which can be presented in two identical ways

$$
\begin{aligned}
\hat{\mathcal{H}}_{\text {Rich }} & =\sum_{l}^{N} z_{l}\left(1+\hat{\sigma}_{l}^{3}\right)-\frac{\lambda}{4} \sum_{l, l^{\prime}} \hat{\sigma}_{l}^{\dagger} \hat{\sigma}_{l^{\prime}}^{-} \\
& \equiv-\sum_{l}\left(2 z_{l} \hat{H}_{l}^{R}-z_{l}+\frac{\lambda}{4}\right)+\lambda\left(\sum_{l} \hat{H}_{l}^{R}\right)^{2}
\end{aligned}
$$

where $\hat{\sigma}^{3, \pm}$ are Pauli matrices, and the operators $\hat{H}_{l}^{R}=-\hat{\sigma}_{l}^{3} / 2+\lambda \hat{H}_{l}^{G}$ represent $N$ mutually commuting $\left[\hat{H}_{l}^{R}, \hat{H}_{l^{\prime}}^{R}\right]=0$ conserved "currents." Note that $\sum_{l} \hat{H}_{l}^{G}=0$, and hence the second term in Eq. (7) can be simplified as $\sum_{l} \hat{H}_{l}^{R}=-\sum_{l} \hat{\sigma}_{l}^{3} / 2$ to give the "total pseudo-spin magnetization," which separates the Hilbert space into sectors with different numbers of Cooper pairs, which were actually studied in Ref. 7 .

Note that the Gaudin model is closely related to the Richardson model (6/7) and corresponds to its infinite coupling limit. It is interesting to see what perturbed WZNW model, would correspond to the KZ equations (2) with the operators $\hat{H}_{l}$ replaced with $\hat{H}_{l}^{R}$. This is a key question addressed in this paper, but in the interest of practical applications, we shall consider a more general form of "new" operators:

$$
\begin{aligned}
& \hat{\tilde{H}}_{l}[\mathcal{C}]=-\hat{U}[\mathcal{C}] \partial_{z_{l}} \hat{U}^{-1}[\mathcal{C}]+\lambda \hat{\tilde{H}}_{l}^{G}[\mathcal{C}], \quad \text { where } \\
& \hat{\tilde{H}}_{l}^{G}[\mathcal{C}]=\frac{1}{k+2} \hat{U}[\mathcal{C}] \hat{H}_{l}^{G} \hat{U}^{-1}[\mathcal{C}] .
\end{aligned}
$$

Here $\hat{\tilde{H}}_{l}^{G}[\mathcal{C}]$ is a rotated Gaudin Hamiltonian, with $\hat{U}[\mathcal{C}]=\exp \left\{\sum_{i} q\left(z_{i}\right) \hat{S}_{i}^{3} \Theta\left[\mathcal{C}, z_{i}\right]\right\}, \mathcal{C}$ is a closed contour in the complex plane, $q(z)$ is an arbitrary analytic function inside $\mathcal{C}$, and $\Theta[\mathcal{C}, z]=1$ if $z$ lies within the region enclosed by the contour and zero otherwise. We emphasize that Eq. (7) contains the conserved "currents" from the Richardson pairing model (6) in the simplest special case, of $k=1$ (i.e., $\hat{\mathbf{S}}$ become Pauli matrices $\hat{\boldsymbol{\sigma}} / 2$ ), $\mathcal{C} \rightarrow \mathcal{C}_{\infty}$ (i.e., the contour $\mathcal{C}_{\infty}$ encloses all points in $\mathbb{C}$ ), and $q(z)=-z / \lambda$, so that $\hat{U}_{\text {Rich }}=\exp \left\{-\sum_{i} z_{i} \hat{\sigma}_{i}^{3} /(2 \lambda)\right\}$.

We first present the main result for the boundary WZNW model, corresponding to operators $\hat{\tilde{H}}_{l}[\mathcal{C}]$ defined in Eq. (77),

$$
S_{\mathrm{BWZNW}}[\mathcal{C}]=S_{\mathrm{WZNW}}+S_{\text {bound }}^{L}[\mathcal{C}]+S_{\text {bound }}^{R}[\overline{\mathcal{C}}],
$$

where $S_{\text {WZNW }}$ is the standard WZNW action (10),

$$
S_{\text {bound }}^{L}[\mathcal{C}]=-\oint_{C} d z q(z) J^{3}(z)
$$

is the "left" boundary term, and the "right" boundary term, $S_{\text {bound }}^{R}[\overline{\mathcal{C}}]$ is given by (9) with $z \rightarrow \bar{z}$ and $J^{3}(z) \rightarrow \bar{J}^{3}(\bar{z})$. In Eq. (9), $J^{3}(z)$ is a component of the "left" current in the $S U(2)$ WZNW theory, defined in a standard way: $J^{a}(z)=(k / 2) \operatorname{tr}\left[\hat{S}^{a} g(z, \bar{z}) \partial_{z} g^{\dagger}(z, \bar{z})\right]$, $a= \pm, 3$. Note that due to conformal invariance the "left" currents do not depend on $\bar{z}$ and likewise the "right" currents, $\bar{J}^{a}(\bar{z})$ do not depend on $z$. Note that the term (9) breaks translational invariance of the model and hence can be interpreted as a generalized impurity ${ }^{2}$. Below we prove that the boundary action gives rises to generalized KZ Eqs. (2) and present exact results for the corresponding correlation functions.

\section{DERIVATION OF THE BOUNDARY WZNW ACTION}

We are seeking to prove that a correlation function of arbitrary primary fields ${ }^{20}$ in the $S U(2)$ boundary WZNW model (8),

$$
\begin{aligned}
G\left(z_{1}, \cdots z_{N}\right) & =\left\langle\phi_{s_{1}}\left(z_{1}\right) \ldots \phi_{s_{N}}\left(z_{N}\right)\right\rangle_{S_{\mathrm{BWZNW}}} \\
& \equiv\left\langle\Phi[\mathcal{C}] \phi_{s_{1}}\left(z_{1}\right) \cdots \phi_{s_{N}}\left(z_{N}\right)\right\rangle_{S_{\mathrm{WZNW}}}
\end{aligned}
$$

satisfies the generalized $\mathrm{KZ}$ equations (2) with operators (7). Here, $\Phi[\mathcal{C}]=e^{-S_{\text {bound }}(\mathcal{C})}$ and $s_{i}$ stands for the spin, $0 \leq s_{i} \leq(k / 2), i=1 \ldots N$.

To solve the $\mathrm{KZ}$ equations (2) we look for $\Phi[\mathcal{C}]$ in the form (9) $\Phi[\mathcal{C}]=e^{\oint_{C} d z q(z) J^{3}(z)}$, where $q(z)$ is an analytic and differentiable function in $\mathcal{C}$, and utilize the two standard key ingredients of the $S U(2)$ WZNW theory and CFTs20: (i) The crux here is the operator product expansion satisfied by the currents with the same chiralities (currents with different chiralities commute); (ii) Action of the Virasoro generators on primary fields. Then the expression for the correlation function $G\left(z_{1}, \cdots z_{N}\right)$ can be simplified by contracting $J^{z}(z)$ in $\Phi[\mathcal{C}]=\sum_{p}(1 / p !)\left(\oint_{\mathcal{C}} d z q(z) J^{3}(z)\right)^{p}$ with all primary fields

$G\left(z_{1}, \cdots z_{N}\right)=\left\langle e^{\sum_{i} q\left(z_{i}\right) \hat{S}_{i}^{3} \Theta\left[\mathcal{C}, z_{i}\right]} \phi_{s_{1}}\left(z_{1}\right) \cdots \phi_{s_{N}}\left(z_{N}\right)\right\rangle$,

where the functional averaging with respect to the $S_{\text {WZNW }}$ is understood. Using the standard technique of Ref. 4 and taking into account the boundary operator we find that indeed the following identity holds

$$
\left[\partial_{z_{i}}-q^{\prime}\left(z_{i}\right) \hat{S}_{i}^{3} \Theta\left[\mathcal{C}, z_{i}\right]-\hat{\tilde{H}}_{i}^{G}[\mathcal{C}]\right] G\left(z_{1}, \cdots z_{N}\right)=0,
$$

where $\hat{\tilde{H}}_{i}^{G}[\mathcal{C}]$ is the rotated Gaudin Hamiltonian defined in (7). Note that if $q(z) \equiv-z / k \lambda$ and all $z_{i}(i=1 \ldots N)$ are inside $\mathcal{C}$, Eq. (11) precisely reproduces modified KZ equations (2) with the Gaudin integrals of motion replaced with those of the Richardson model with the interaction parameter $\lambda$. Hence, we recover the amazing fact that the correlation functions of the boundary 
WZNW model carry information about the exact correlation functions of the pairing model.

\section{SOLUTION OF THE KNIZHNIK-ZAMOLODCHIKOV EQUATIONS}

We now show that the generalized KZ Eqs. (2) can be solved exactly using the standard off-shell Bethe Ansatz technique ${ }^{21}$. This method applies if all $\hat{\tilde{H}}_{l}$ are commuting, in what follows we will consider the case when all $z_{i} \in \mathcal{C}$. Look for the solution in an integral form

$G\left(z_{1} \cdots z_{N}\right)=\oint \prod_{k=1}^{M} d u_{k} \chi\left(\left\{u_{\alpha}\right\} \mid\left\{z_{i}\right\}\right) \mathcal{V}\left(\left\{u_{\alpha}\right\} \mid\left\{z_{i}\right\}\right)$

where $M$ is fixed from the condition that the correlation function $G\left(z_{1} \cdots z_{N}\right)$ should be a singlet with respect to the global $S U(2): \hat{S}^{3} G\left(z_{1} \cdots z_{N}\right) \equiv 0$ for WZNW model $\underline{4}$ and also for our boundary case. The integrations are to be taken here over canonical cycles in the $\mathrm{n}$ dimensional complex space where points $z_{i}$ are excluded, with coefficients, defined by the monodromy group of the function $\chi\left(\left\{u_{\alpha}\right\},\left\{z_{i}\right\}\right)$. Then it follows that $M=\sum_{i=1}^{N} s_{i}$. We look for eigenstates of a set of commuting Hamiltonian operators, $\hat{H}_{i}^{R}$, of the pairing model in the form

$$
\mathcal{V}\left(u_{1} \cdots u_{M} \mid\left\{z_{i}\right\}\right)=\hat{S}^{\dagger}\left(u_{1}\right) \cdots \hat{S}^{\dagger}\left(u_{M}\right)|0\rangle
$$

where

$$
\hat{S}^{\dagger}(u)=\sum_{i=1}^{N} \frac{\hat{S}_{i}^{\dagger}}{u-z_{i}}
$$

and the bare vacuum state, $|0\rangle$, is a direct product of lowest weight vectors of the corresponding representation $s_{i}: \hat{S}_{i}^{3}\left|s_{i}, m_{i}\right\rangle=m_{i}\left|s_{i}, m_{i}\right\rangle$, where $m_{i}=-s_{i}$. For example if $k=1$ and all primary fields in $G\left(z_{1} \cdots z_{N}\right)$ are from $s_{i}=1 / 2$ representation space of $\mathrm{SU}(2)$, then $M=N / 2$ and $|0\rangle=\left(\begin{array}{l}0 \\ 1\end{array}\right)_{1} \otimes \ldots \otimes\left(\begin{array}{l}0 \\ 1\end{array}\right)_{N}$. In the basis where the primary fields are defined by spin $s$ and its z-projection, $m=-s \ldots s$, their correlation function $\left\langle\phi_{s_{1}}^{m_{1}}\left(z_{1}\right) \cdots \phi_{s_{N}}^{m_{N}}\left(z_{N}\right)\right\rangle$ is connected to the general expression (12) as follows

$$
\begin{aligned}
& \left\langle\phi_{s_{1}}^{m_{1}}\left(z_{1}\right) \cdots \phi_{s_{N}}^{m_{N}}\left(z_{N}\right)\right\rangle \\
= & \left\langle s_{N}, m_{N}\right| \cdots\left\langle s_{1}, m_{1}\right| G\left(z_{1} \cdots z_{N}\right),
\end{aligned}
$$

where $\left\langle s_{i}, m_{i}\right| \hat{S}_{i}^{3}=\left\langle s_{i}, m_{i}\right| m_{i}$. We found that $\chi\left(\left\{u_{\alpha}\right\} \mid\left\{z_{i}\right\}\right)$ has the following form

$$
\begin{gathered}
\chi\left(\left\{u_{\alpha}\right\} \mid\left\{z_{i}\right\}\right)=\chi_{0}\left(\left\{u_{\alpha}\right\} \mid\left\{z_{i}\right\}\right) \\
\times \exp \left\{\frac{1}{\lambda(k+2)}\left[-\lambda k \sum_{i=1}^{N} m_{i} q\left(z_{i}\right)+\sum_{\alpha} u_{\alpha}\right]\right\},
\end{gathered}
$$

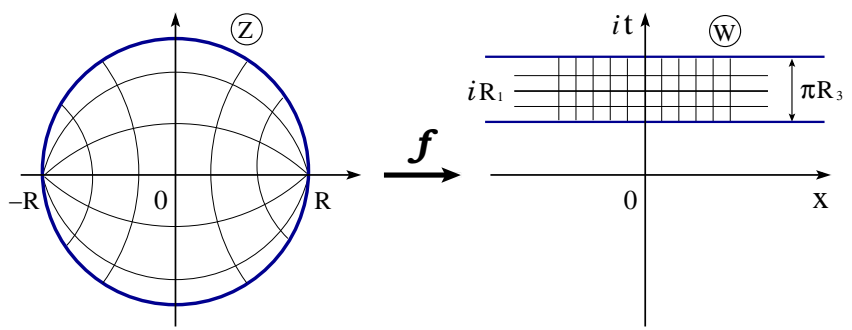

FIG. 1: (Color online) An example of a conformal map. Function $w=f(z)=i R_{1}+R_{2}+R_{3} \log \left(\frac{z-R}{z+R}\right)$ maps a disc with radius $R$ to a strip of width $\pi R_{3}$ centered at $\operatorname{Im}(w)=R_{1}$.

where

$$
\begin{aligned}
\chi_{0}\left(\left\{u_{\alpha}\right\} \mid\left\{z_{i}\right\}\right) & =\prod_{i \neq j}\left(z_{i}-z_{j}\right)^{\frac{m_{i} m_{j}}{(k+2)}} \prod_{\beta \neq \alpha}\left(u_{\alpha}-u_{\beta}\right)^{\frac{1}{(k+2)}} \\
& \times \prod_{\gamma, i}\left(u_{\gamma}-z_{i}\right)^{\frac{-m_{i}}{(k+2)}}
\end{aligned}
$$

is the known solution ${ }^{21,22}$ to the KZ Eqs. for the canonic $S U(2)$ WZNW model. In general these solutions can be expressed analytically in terms of multi-variable confluent hypergeometric functions ${ }^{23,24}$. Note that when $\lambda \rightarrow \infty$, the Richardson pseudospin model reduces to the Gaudin magnet, and consequently $\chi$ in Eq. (16) reduces to $\chi_{0}$. Moreover, analysis of Eqs. (12) at $q(z) \equiv-z / k \lambda$ suggests that the integral over $u_{1} \ldots u_{M}$ in Eq. (12) has a saddle point defined by the condition

$$
\frac{1}{\lambda}+\sum_{\alpha \neq \beta}^{M} \frac{1}{u_{\alpha}-u_{\beta}}+\sum_{i=1}^{N} \frac{s_{i}}{u_{\beta}-z_{i}}=0
$$

Interestingly, this condition coincides with Richardson equations for the eigenvalues of the reduced BCS Hamiltonian (6).

\section{BOSONIZED ACTION AT $k=1$ : IMPLICATIONS}

The WZNW model (8) at $k=1$ can also be realized as a free boson theory with central charge $c=1^{22}$. Following the standard bosonization technique we introduce a scalar field, $\hat{\varphi}=\hat{\varphi}(z)+\hat{\bar{\varphi}}(\bar{z})$, and rewrite the z-component of the current in the form $J^{3}(z)=\frac{i}{\sqrt{2 \pi}} \partial_{z} \hat{\varphi}(z)$. Note that this representation of $J^{z}$ is correct only locally. The full action (1) at $k=1$ reads

$S=\frac{1}{4 \pi} \int d z d \bar{z} \partial_{z} \varphi \partial_{\bar{z}} \varphi+\frac{i}{\lambda \sqrt{2 \pi}}\left[\oint_{\mathcal{C}} d z z \partial_{z} \varphi+\right.$ a. c. $]$

where a. c. stands for an antiholomorphic contribution. We note that this bosonized version of the $k=1$ action was discussed earlier in Refs. 13, 14 by Sierra.

Here we emphasize that due to the presence of the boundary term defined by an arbitrary contour $\mathcal{C}$ and 
conformal invariance of the WZNW model make the boundary WZNW model a very useful tool to classify and study low-energy, strong coupling disordered quantum systems 25 as well as various systems driven out of equilibrium. Let us use bosonic version of the $k=1$ theory as an illustrative example. If all $z_{i}$ are real, we have a standard physical Richardson pseudo-spin model. Here contour $\mathcal{C}$ can be chosen as boundary of a narrow strip encompassing all $z_{i}$, which explicitly shows that we have an equilibrium system. On contrary, if some of $z_{i}$ have nonzero imaginary part, the contour can be a circle with radius $R$. In this case parametrization $z=i t+x$, where $t$ is the dimensionless time and $x$ is the dimensionless coordinate, is inconvenient, as we generate a complicated time dependent term in our bosonic Hamiltonian. Interestingly enough, this, from a first sight abstract problem is closely related to another, well defined and physically motivated system. Consider the conformal map, $z \rightarrow w$, where $w=i t+x$, which transforms a disc with radius $R$ to an infinite strip, see Fig. 1. The bulk action is invariant under such transformations, while the boundary term will transform into $\sim \oint_{\mathcal{C}^{\prime}} d w \sinh ^{-2}\left(\frac{w-i R_{1}-R_{2}}{2 R_{3}}\right) \varphi[z(w)]$. Here contour $\mathcal{C}^{\prime}$ is the boundary of a strip of width $\pi R_{3}$ centered at $t_{0}=R_{1}$. This term contributes to the Hamiltonian and makes it time dependent. It describes a single instantaneous perturbation on the system at $t=t_{0}$, which however does not brake integrability. Remarkably, we can extract enormous information about physical properties of such systems by analyzing exact correlation functions.

\section{PRACTICAL APPLICATIONS OF THE BOUNDARY WZNW MODEL}

Boundary action Eq. (9) together with the expression (16) for the correlation functions represent our main mathematical result. As argued, it has important consequences for a variety of seemingly unrelated physical models, notably dynamical systems. We provide here an explicit example of such correspondence between Maxwell-Bloch (MB) theory of a two-level laser ${ }^{26}$, which is shown to map onto the BWZNW model. Below, we derive for the first time an exact solution to the system of MB equations with damping

$$
\begin{aligned}
& \partial_{\eta} \mathcal{E}+\gamma \mathcal{E}=\mathcal{P} \\
& \partial_{\xi} \mathcal{P}+\gamma_{\perp} \mathcal{P}=\mathcal{N} \mathcal{E} \\
& \partial_{\xi} \mathcal{N}+\frac{1}{2}\left(\mathcal{E} \mathcal{P}^{*}+\mathcal{E}^{*} \mathcal{P}\right)=-\gamma_{||} \mathcal{N}+\mathcal{N}_{0}
\end{aligned}
$$

where $\mathcal{E}(\eta, \xi)$ is the complex electrical field amplitude, $\mathcal{P}(\eta, \xi)$ is the polarization of the medium, $\mathcal{N}(\eta, \xi)$ is the population inversion, and $\eta=\Omega x / c$ and $\xi=\Omega(t-x / c)$ are given in terms of real space, $x$, and time, $t$, with $\Omega$ being a physical constant that depends on material and cavity medium, and $c$ is the speed of light. In Eq. (20), $\gamma \geq 0$ is a decay rate of energy losses inside the laser medium and the constants $\gamma_{\perp}$ and $\gamma_{\|}$are damping coefficients of medium polarization and population inversion. Dissipation in the population inversion equation tends to return $\mathcal{N}$ to $\mathcal{N}_{0} / \gamma_{||}$, which is determined by the pumping.

Amazingly, the Hamiltonian formulation of (20) for $\gamma_{\|}=\gamma_{\perp}=\gamma=0$ reduces to a set of $\mathrm{KZ}$ equations (11) with linear $q(z) \equiv \alpha z^{27,28}$. We show now that the BWZNW model with $q(z) \equiv \alpha z+\beta z^{2}$ and the corresponding generalized KZ Eqs. (11) describe the system (20) with finite damping parameters $\gamma, \gamma_{\perp} \geq 0$. First, we observe by analogy with Ref. [27] that the set of MB equations (20) with damping and pumping can be obtained from the compatibility condition of the following system of liner differential equations with complex spectral parameter $z \in \mathbb{C}$

$$
\partial_{\xi} \psi=\left[\left(z-\frac{\gamma_{\perp}}{2}\right) \sigma_{3}+U_{0}\right] \psi
$$

and

$$
\left(\partial_{\eta}+\frac{\mathcal{N}_{0}}{z} \partial_{z}\right) \psi=\left(\frac{\hat{\rho}}{4 z}-\frac{\gamma}{2} \sigma_{3}\right) \psi
$$

where

$$
U_{0}=\frac{1}{2}\left(\begin{array}{cc}
0 & \mathcal{E} \\
-\mathcal{E}^{*} & 0
\end{array}\right), \quad \hat{\rho}=\frac{1}{2}\left(\begin{array}{cc}
\mathcal{N} & -\mathcal{P} \\
-\mathcal{P}^{*} & -\mathcal{N}
\end{array}\right) .
$$

According to the method of isomonodromy solutions of differential equations, $\stackrel{28,29}{,}$ variety of solutions of the MB equations can be obtained by classifying solutions of an auxiliary equation, $\partial_{z} \psi(z)=A(z, \xi, \eta) \psi(z)$, that are consistent with the original MB equations. We found that consistent with (21) choice of $A(z, \xi, \eta)$, which produces $N$-soliton solutions of $\mathrm{MB}$ equations in the presence of pumping and damping, reads

$$
\begin{aligned}
A(z, \xi, \eta) & =\left(\xi-\xi_{0}\right) \sigma_{3}+\sum_{j=1}^{N} \frac{A_{j}}{z-z_{j}} \\
z_{j} & =\sqrt{2 N_{0} \eta-k_{j}^{2}}
\end{aligned}
$$

with parameters $k_{j}^{2}, \xi_{0} \in \mathbb{R}$. Substituting expression for $\partial_{z} \psi(z)$ together with Eq. (24) into Eqs. (21), writing compatibility conditions and equating the residues of the poles at $z=z_{i}, i=1 \ldots N$, one will obtain for functions $A_{j}$ :

$$
\begin{aligned}
\partial_{\xi} A_{j} & =\left[\left(z_{j}-\frac{\gamma_{\perp}}{2}\right) \sigma_{3}+U_{0}, A_{j}\right], \\
\partial_{\eta} A_{j} & =\left[\left(\frac{\hat{\rho}}{4 z_{j}}-\frac{\gamma}{2}\right) \sigma_{3}+U_{0}, A_{j}\right]
\end{aligned}
$$

where

$$
\begin{aligned}
U_{0} & =\frac{1}{\xi-\xi_{0}}\left(\sum_{l=1}^{N} A_{l}-\operatorname{diag} \sum_{l=1}^{N} A_{l}\right), \\
\hat{\rho} & =4 N_{0}\left(\left(\xi-\xi_{0}\right) \sigma_{3}-\sum_{l=1}^{N} \frac{A_{l}}{z_{l}}\right) .
\end{aligned}
$$


Eqs. 25) admit a Hamiltonian structure with the Poisson brackets 28

$$
\left\{\left(A_{m}\right)_{a b},\left(A_{n}\right)_{c d}\right\}=\delta_{m n}\left(\left(A_{m}\right)_{a d} \delta_{b c}-\delta_{a d}\left(A_{m}\right)_{b c}\right),
$$

which corresponds to the $\operatorname{sl}(2)$ algebra on a chain. Therefore, Eqs. (25) acquire the form $\partial_{\xi} A_{j}=$ $\left\{A_{j}, H_{\xi}\right\}, \quad \partial_{\eta} A_{j}=\left\{A_{j}, H_{\eta}\right\}$, with Hamiltonian operators

$$
\begin{aligned}
& H_{\xi}=\sum_{k=1}^{N}\left(z_{k}-\frac{\gamma_{\perp}}{2}\right) \operatorname{tr}\left(A_{k} \sigma_{3}\right)+\frac{S^{-} S^{+}+S^{+} S^{-}}{\xi-\xi_{0}}, \\
& H_{k}=\sum_{k=1}^{N}\left(\frac{\left(\xi-\xi_{0}\right)}{z_{k}}-2 \gamma\right) \operatorname{tr}\left(A_{k} \sigma_{3}\right)+\sum_{j=1}^{N} \frac{\operatorname{tr}\left(A_{k} A_{j}\right)}{z_{k}\left(z_{k}-z_{j}\right)},
\end{aligned}
$$

and $S^{+,-}=\left(\sum_{k=1}^{N} A_{k}\right)_{12,21}$.

Quantization of the MB system implies replacement of Poisson brackets by commutators, $\{,\} \rightarrow[$,$] , and intro-$ duction of a quantum wave function, $\Psi\left(\xi,\left\{z_{k}\right\}\right)$. Then the $s l(2)$ algebra (27) acquires the following matrix realization

$$
A_{k}=i\left(\begin{array}{cc}
S_{k}^{3} & S_{k}^{+} \\
S_{k}^{-} & -S_{k}^{3}
\end{array}\right),
$$

which, together with transformation $\xi \rightarrow i \xi$, leads to the set of Hamiltonian operators corresponding to (20):

$$
\begin{aligned}
& \hat{h}_{\xi}=i^{2} \sum_{k=1}^{N}\left(z_{k}-\frac{\gamma_{\perp}}{2}\right) \hat{S}_{k}^{3}+i^{2} \frac{\hat{S}^{-} \hat{S}^{+}+\hat{S}^{+} \hat{S}^{-}}{\xi-\xi_{0}}, \\
& \hat{h}_{k}=i^{2}\left[\left(\xi-\xi_{0}\right)-2 \gamma z_{k}\right] \hat{S}_{k}^{3}-i^{2} \sum_{j \neq k}^{N} \frac{\hat{\mathbf{S}}_{k} \hat{\mathbf{S}}_{j}}{z_{k}-z_{j}}
\end{aligned}
$$

where $k=1 \ldots N$, and $\hat{S}^{ \pm}=\sum_{k} \hat{S}_{k}^{ \pm}$. On a quantum level, the "wave function," $\Psi\left(\xi, z_{1} \ldots z_{N}\right)$, satisfying the set of "multitime," $t \rightarrow\left(\xi, z_{1} \ldots z_{N}\right)$, Schrödinger equations,

$$
\begin{aligned}
i \partial_{\xi} \Psi & =\hat{h}_{\xi} \Psi, \\
i \partial_{z_{k}} \Psi & =\hat{h}_{k} \Psi,
\end{aligned}
$$

unambiguously determine the solution of MB equations. As we see, the second set of Schrödinger equations coincides with generalized KZ equations (11) with the following parameters: $k+2=1, i\left(\xi-\xi_{0}\right)=1 / \lambda, q(z)=$ $-z / \lambda-\gamma z^{2}$, and $\mathcal{C}=\mathcal{C}_{\infty}$, which encompasses all $z_{j}=$ $\left(2 \mathcal{N}_{0} \eta-k_{j}^{2}\right)^{1 / 2}$, where $k_{j}^{2}, \xi_{0} \in \mathbb{R}$ are free parameters. The first equation is formally an ordinary Schrödinger equation with "time," $\xi=\Omega(t-x / c)$. Therefore, this maps the problem onto a dynamical boundary WZNW model, with the boundary action $S_{M B}=\oint_{\mathcal{C}_{\infty}} d z q(z) J^{3}(z)$, correlation functions of which depend on an additional parameter, $\xi$, playing the role of time.

By analogy with Eq. (12), solution for $\Psi\left(\xi, z_{1} \ldots z_{N}\right)$ is then found to be

$$
\begin{aligned}
\Psi\left(\xi, z_{1} \cdots z_{N}\right) & =\oint \prod_{k=1}^{M} d u_{k} \chi_{M B}\left(\left\{u_{\alpha}\right\} \mid\left\{z_{i}\right\}\right) \\
& \times \mathcal{V}\left(\left\{u_{\alpha}\right\} \mid\left\{z_{i}\right\}\right),
\end{aligned}
$$

with

$$
\begin{aligned}
& \frac{\chi_{M B}\left(\left\{u_{\alpha}\right\} \mid\left\{z_{i}\right\}\right)}{\chi_{0}\left(\left\{u_{\alpha}\right\} \mid\left\{z_{i}\right\}\right)}=\left(\xi-\xi_{0}\right)^{i\left(\sum m_{i}-M\right)} e^{2 i\left(\xi-\xi_{0}\right) \sum z_{i} m_{i}} \\
& \times \exp \left\{\frac{1}{\lambda}\left[\sum_{i=1}^{N} m_{i}\left(\frac{\gamma_{\perp}}{2}-\lambda q\left(z_{i}\right)\right)+\sum_{\alpha} u_{\alpha}\right]\right\} .
\end{aligned}
$$

Eqs. (331) and (32) determine the form of the wave function of quantum states in the quantized MB system. Importantly, this wave function is cardinally different from the correlation function of primary fields in the bulk WZNW model. This is because the expression in the right-hand-side of Eq. (33) must be integrated in Eq. (32) together with $\chi_{0}$, while only the later appears in the WZNW model.

In order to find the solution of the system of classical MB equations (31), one should average the angular momentum operators, $S^{ \pm}, S^{3}$, with respect to the wave functions (33). Then the solution of classical MB equations for physical quantities $\mathcal{E}, \mathcal{P}$, and $\mathcal{N}$ can be found from Eqs. (23), (25), and (26) as follows:

$$
\begin{aligned}
& \mathcal{E}^{2}=\left\langle\Psi^{*}\left|\frac{\kappa^{2}}{\left(\xi-\xi_{0}\right)^{2}} \sum_{j=1}^{N} \hat{S}_{j}^{-} \sum_{j=1}^{N} \hat{S}_{j}^{+}\right| \Psi\right\rangle, \\
& \mathcal{P}^{2}=64 \mathcal{N}_{0}^{2} \kappa\left\langle\Psi^{*}\left|\sum_{j=1}^{N} \frac{\hat{S}_{j}^{-}}{z_{j}} \sum_{j=1}^{N} \frac{\hat{S}_{j}^{+}}{z_{j}}\right| \Psi\right\rangle, \\
& \mathcal{N}=8 \mathcal{N}_{0}\left\langle\Psi^{*}\left|\left[\xi-\xi_{0}-\kappa \sum_{j=1}^{N} \frac{\hat{S}_{j}^{3}}{z_{j}}\right]\right| \Psi\right\rangle .
\end{aligned}
$$

These equations provide $N$-soliton solutions to the MB system. In general these solutions have compact integral representations which can be evaluated and compared with other numerical ${ }^{30}$ and experimental ${ }^{31}$ data. In Appendix we evaluate this integral for $N=2$ soliton case and express the solution for $\mathcal{E}, \mathcal{P}$, and $\mathcal{N}$ in terms of known Kummer confluent hypergeometric functions.

\section{CONCLUSION}

In conclusion, this work has introduced a method of reverse construction of boundary WZNW models from the generalized Knizhnik-Zamolodchikov equations satisfied by the exact correlation functions and demonstrated the application of this method on the explicit example of KZ equations with conserved currents of the Richardson model. Thereby, we established a direct connection between the discrete pairing model of superconductivity and the boundary WZNW model, which we identified and solved. We have established that the solutions of modified KZ equations are defined by the off-shell states of the Richardson model. Our construction is close in spirit but technically different from the BCS/CFT correspondence discussed earlier by Sierra ${ }^{13}$. Our main moti- 
vation has been to precisely identify the boundary operator in the WZNW model, which is related to the Richardson-type models. Our other motivation has been to outline a range of practical applications of the discovered correspondence, which is argued to be very wide and includes a variety of dynamical systems that can be mapped on the BWZNW theories and solved exactly in many cases. One such mapping and solution for the dynamical system describing radiation of a two level laser with pumping and damping was presented. Dynamic properties of the laser were computed exactly exploiting the integrability of this latter system.

Acknowledgements - This research was supported by the NSF CAREER award, DMR-0847224.

\section{APPENDIX}

Here we derive the analytical expression of the twosoliton solution of $\mathrm{MB}$ equations, when $N=2$ and $M=1$. For simplicity we consider the case with finite pumping, $\mathcal{N}_{0}$, and medium polarization damping, $\gamma_{\perp}$, coefficients, but with $\gamma=0$. In this case we have one integration parameter $u$ and two parameters, $z_{1}, z_{2}$. Then from Eqs. (13) and (14) it follows that

$$
\begin{aligned}
\mathcal{V}(u) & =\sum_{i=1}^{2} \frac{\hat{S}_{i}^{\dagger}}{u-z_{i}}|\downarrow, \downarrow\rangle \\
& =\frac{1}{u-z_{1}}|\uparrow, \downarrow\rangle+\frac{1}{u-z_{2}}|\downarrow, \uparrow\rangle .
\end{aligned}
$$

In order to construct the wave function (32) and (33), we should integrate $\mathcal{V}(u)$ together with the term corresponding to $\chi_{0},\left[\left(u-z_{1}\right)\left(u-z_{2}\right)\right]^{-1 / 2(k+2)}$, over $u$ along a contour surrounding the brunch-cut at $\left(z_{1}, z_{2}\right)$ [see Eq. (12) and the discussion]. To perform the resulting integration, we make use of the identity

$$
\begin{aligned}
& \oint_{C} d u\left(u-z_{1}\right)^{-a}\left(u-z_{2}\right)^{-1+a} e^{-b u} \\
= & B(a, 1-a)_{1} F_{1}\left[1-a ; 1 ; b\left(z_{2}-z_{1}\right)\right]
\end{aligned}
$$

where ${ }_{1} F_{1}$ is the Kummer confluent hypergeometric function and $B(a, 1-a)$ is the Beta-function 32 . Then for $a=1 / 2$ and $b=1 / \lambda$ we have

$$
{ }_{1} F_{1}\left[\frac{1}{2} ; 1 ; \frac{\left(z_{1}-z_{2}\right)}{\lambda}\right]=e^{\frac{z}{2 \lambda}} I_{0}\left[\frac{\left(z_{1}-z_{2}\right)}{2 \lambda}\right],
$$

with $I_{0}$ being the modified Bessel function of zero order. Now, returning to the real time, $\xi \rightarrow-i \xi$, by analytic continuation, and keeping causal behavior of the wave function, $\Psi$, we will have

$$
\begin{aligned}
\Psi & =\pi\left(\xi-\xi_{0}\right)^{-i} e^{-\left|\xi-\xi_{0}\right| \frac{\gamma_{1}}{2}-\left|\left(\xi-\xi_{0}\right)\left(z_{1}-z_{2}\right)\right|}\left(\left(z_{1}-z_{2}\right)^{-\frac{1}{4}} e^{\frac{i\left(\xi-\xi_{0}\right)\left(z_{2}-z_{1}\right)}{2}} I_{0}\left[\frac{i\left(\xi-\xi_{0}\right)\left(z_{2}-z_{1}\right)}{2}\right]|\uparrow, \downarrow\rangle\right. \\
& \left.+\left(z_{2}-z_{1}\right)^{-\frac{1}{4}} e^{\frac{i\left(\xi-\xi_{0}\right)\left(z_{1}-z_{2}\right)}{2}} I_{0}\left[\frac{i\left(\xi-\xi_{0}\right)\left(z_{1}-z_{2}\right)}{2}\right]|\downarrow, \uparrow\rangle\right) .
\end{aligned}
$$

According to Eqs. (34), to find $\mathcal{E}$ one should act by the operator $S_{1}^{+}+S_{2}^{+}$on the expression (38) for $\Psi$ and calculate the norm. By doing so and after some simple algebra we obtain

$$
\begin{aligned}
\mathcal{E} & =\frac{\pi}{\left|\xi-\xi_{0}\right|} e^{-\left|\xi-\xi_{0}\right| \frac{\gamma_{1}}{2}-\left|\left(\xi-\xi_{0}\right)\left(z_{1}-z_{2}\right)\right|} \operatorname{Abs}\left[\left(z_{1}-z_{2}\right)^{-\frac{1}{4}} e^{\frac{i\left(\xi-\xi_{0}\right)\left(z_{2}-z_{1}\right)}{2}} I_{0}\left[\frac{i\left(\xi-\xi_{0}\right)\left(z_{2}-z_{1}\right)}{2}\right]\right. \\
& \left.+\left(z_{2}-z_{1}\right)^{-\frac{1}{4}} e^{\frac{i\left(\xi-\xi_{0}\right)\left(z_{1}-z_{2}\right)}{2}} I_{0}\left[\frac{i\left(\xi-\xi_{0}\right)\left(z_{1}-z_{2}\right)}{2}\right]\right]
\end{aligned}
$$

where the notion Abs means absolute value. Similarly, the expressions for the polarization of the medium $\mathcal{P}$ and the population inversion $\mathcal{N}$ read:

$$
\begin{aligned}
\mathcal{P} & =8 \pi \mathcal{N}_{0} e^{-\left|\xi-\xi_{0}\right| \frac{\gamma \perp}{2}-\left|\left(\xi-\xi_{0}\right)\left(z_{1}-z_{2}\right)\right|} \operatorname{Abs}\left[\frac{1}{z_{2}}\left(z_{1}-z_{2}\right)^{-\frac{1}{4}} e^{\frac{i\left(\xi-\xi_{0}\right)\left(z_{2}-z_{1}\right)}{2}} I_{0}\left[\frac{i\left(\xi-\xi_{0}\right)\left(z_{2}-z_{1}\right)}{2}\right]\right. \\
& \left.+\frac{1}{z_{1}}\left(z_{2}-z_{1}\right)^{-\frac{1}{4}} e^{\frac{i\left(\xi-\xi_{0}\right)\left(z_{1}-z_{2}\right)}{2}} I_{0}\left[\frac{i\left(\xi-\xi_{0}\right)\left(z_{1}-z_{2}\right)}{2}\right]\right]
\end{aligned}
$$


and

$$
\begin{aligned}
\mathcal{N} & =8 \pi \mathcal{N}_{0} e^{-\left|\xi-\xi_{0}\right| \frac{\gamma_{1}}{2}-\left|\left(\xi-\xi_{0}\right)\left(z_{1}-z_{2}\right)\right|} \operatorname{Re}\left[\left(\xi-\xi_{0}+\frac{1}{2 z_{2}}-\frac{1}{2 z_{1}}\right)\left(z_{1}-z_{2}\right)^{-\frac{1}{4}} e^{\frac{i\left(\xi-\xi_{0}\right)\left(z_{2}-z_{1}\right)}{2}} I_{0}\left[\frac{i\left(\xi-\xi_{0}\right)\left(z_{2}-z_{1}\right)}{2}\right]\right. \\
& \left.+\left(\xi-\xi_{0}+\frac{1}{2 z_{1}}-\frac{1}{2 z_{2}}\right)\left(z_{2}-z_{1}\right)^{-\frac{1}{4}} e^{\frac{i\left(\xi-\xi_{0}\right)\left(z_{1}-z_{2}\right)}{2}} I_{0}\left[\frac{i\left(\xi-\xi_{0}\right)\left(z_{1}-z_{2}\right)}{2}\right]\right] .
\end{aligned}
$$

We remind the reader that in all expressions above $z_{j}=$ $\sqrt{2 \mathcal{N}_{0} \eta-k_{j}^{2}}, \quad(j=1,2)$, with constant parameters $k_{1}$ and $k_{2}$. Here, $\eta=\Omega x / c, \xi=\Omega(t-x / c)$ with $\Omega$ being a physical constant that characterizes the material and the cavity medium. To the best of our knowledge, Eqs. (39), (40) and (41) have never been derived in the literature before.
1 I. Affleck and F. D. M. Haldane, Phys. Rev. B 36, 5291 (1987).

2 S. Ryu, C. Mudry, A. Ludwig, and A. Furusaki, Nucl. Phys. B 839, 341-376 (2010).

3 A. M. Polyakov and P. B. Wiegmann, Phys. Lett. B 131, 121 (1983); 141, 223 (1983).

4 D. Knizhnik and A. B. Zamolodchikov, Nucl. Phys. B247, 83 (1984).

5 M. Gaudin, J. Phys. 37, 1087 (1976).

6 J. Dukelsky, S. Pittel, and G. Sierra, Rev. Mod. Phys. 76, 643 (2004)

7 R. W. Richardson, Phys. Lett. 3, 277 (1963); R. W. Richardson, J. Math. Phys. 18, 1802 (1977).

8 J. von Delft and D. C. Ralph, Phys. Rep. 345, 61 (2001) and references therein.

9 M. C. Cambiaggio, A. M. F. Rivas, and M. Saraceno, Nucl. Phys. A 624, 157 (1997).

10 L. Amico, G. Falci, and R. Fazio, J. Phys. A 34, 6425 (2001).

11 L. Amico and A. Osterloh, Phys. Rev. Lett. 88, 127003 (2002).

12 J. von Delft and R. Poghossian, Phys. Rev. B 66, 134502 (2002).

13 G. Sierra, Nucl. Phys. B 572, 517 (2000);

14 G. Sierra, Proc. of NATO Workshop on Stat. Field Theories, Como 2001, hep-th/0111114

15 R. Teodorescu, in Leading-edge Superconductivity Research Developments. Editor T. Watanabe. Nova Science Publishers (2008).

16 E. Yuzbashyan, A. Baytin, and B. Altshuler, Phys. Rev. B 71, 094505 (2005); E. Yuzbashyan, Phys. Rev. B 78, 184507 (2008).

17 A. M. Garcia-Garcia, J. D. Urbina, E. A. Yuzbashyan, K. Richter, and B. L. Altshuler, Phys. Rev. Lett. 100, 187001 (2008); arXiv:0911.1559.

18 A. Faribault, P. Calabrese, and J.-S. Caux, Phys. Rev. B 77, 064503 (2008).
19 D.C.Ralph, C.T. Black, and M. Tinkham, Phys. Rev. Lett. 76, 688 (1996); 78, 4087 (1997).

20 A. A. Belavin, A. M. Polyakov, and A. B. Zamolodchikov, Nucl. Phys. B 241, 333 (1984).

21 H. M. Babujian, J. Phys. A 34, 6425 (1994); H. M. Babujian and R. Flume, Mod. Phys. Lett. A 9, 2029 (1994).

22 A. O. Gogolin, A. A. Nersesyan, and A. M. Tsvelik, Bosonization in Strongly Correlated Systems. Cambridge University Press (1999).

${ }^{23}$ K. Aomoto, J. Math. Soc. Japan 39, 191 (1987).

${ }^{24}$ V. A. Vasilev, I. M. Gelfand and A. V. Zelevinski, Func. Anal. Priloz. 21, 19 (1987).

25 P. Fendley and R. M. Konik, Phys. Rev. B 62, 9359 (2000).

26 T. Numai, Fundamentals of Semiconductor Lasers. Springer (2004).

27 S. P. Burtsev, V. E. Zakharov, and A. V. Mikhailov, Theor. Math. Phys. 70, 227 (1987).

28 H. Babujian and A. Kitaev, J. Math. Phys. 39, 2499 (1998).

29 A. R. Its, Math. USSR Izv. 26, 497 (1986).

30 I. R. Al'miev, O. Larroche, D. Benredjem, J. Dubau, S. Kazamias, C. Möller, and A. Klisnick, Phys. Rev. Lett. 99, 123902 (2007); C. M. Kim, J. Lee, and K. A. Janulewicz, Phys. Rev. Lett. 104, 053901 (2010).

31 see e.g. Ph. Zeitoun, G. Faivre, S. Sebban, T. Mocek, A. Hallou, M. Fajardo, D. Aubert, Ph. Balcou, F. Burgy, D. Douillet, S. Kazamias, G. de Lachèze-Murel, T. Lefrou, S. le Pape, P. Mercère, H. Merdji, A. S. Morlens, J. P. Rousseau, and C. Valentin, Nature (London) 431, 426 (2004); B. Cros, T. Mocek, I. Bettaibi, G. Vieux, M. Farinet, J. Dubau, S. Sebban, and G. Maynard, Phys. Rev. A 73, 033801 (2006); J. Costello, Nat. Photon. 2, 67 (2008); Y. Wang, E. Granados, F. Pedaci, D. Alessi, B. Luther, M. Berrill, and J. J. Rocca, Nat. Photon. 2, 94 (2008).

32 I. S. Gradshtein and I. M. Ryzhik, Tables of integrals, series and products, Academic Press, INC (1996). 